\title{
Chromospheric heating by the Farley-Buneman instability
}

\author{
J. M. Fontenla, W. K. Peterson, and J. Harder
}

\author{
Laboratory for Atmospheric and Space Physics (LASP), University of Colorado, Boulder, CO, 80309, USA \\ e-mail: fontenla@lasp.colorado.edu
}

Received 20 August 2007 / Accepted 7 January 2008

\begin{abstract}
Context. Chromospheric heating produces UV emissions that can only occur in an enhanced electron temperature medium. In the quiet Sun the radiative losses are orders of magnitude larger than those in the much hotter corona. Chromospheric heating mechanisms considered previously (e.g. shock waves and nanoflares) have failed to account for the observed persistency and uniformity of UV lines and continua. Also, resistive magnetic free-energy dissipation is not efficient enough in the highly electrically conductive solar atmosphere.

Aims. In this paper we consider plasma effects in the low chromosphere and propose that the Farley-Buneman (hereafter FB) plasmainstability mechanism provides the mechanism for dissipating the energy of convectively driven motions of neutral atoms into chromospheric heating in the Sun and other cool stars that have a partially ionized chromosphere.

Methods. Analysis of the ion acoustic sound speed and consideration of recent measurements of magnetic field in the quiet, internetwork, solar low chromosphere are carried out in the context of understanding the characteristics and onset of chromospheric heating. The FB instability is triggered by the cross-field motion of the partially ionized gas at velocities in excess of the ion acoustic velocity. The ions acquire their cross-field velocities through collisions with the much denser chromospheric neutral atoms. Estimates of cross-field velocities are obtained from consideration of both spectral line widths and convection numerical simulations that indicate values from a few to several $\mathrm{km} \mathrm{s}^{-1}$ at the top of the practically radiative-equilibrium low chromosphere.

Results. The FB instability is triggered by the cross-field motion of the neutral component of the partially ionized gas at velocities in excess of the ion acoustic velocity. This instability occurs in the solar chromosphere because electrons become strongly magnetized just above the photosphere, while heavy ions and protons remain unmagnetized, and only at the very top of the chromosphere do they become magnetized.

Conclusions. We find that convective overshoot motions are drivers of the FB instability and provide enough energy to account for the upper chromospheric radiative losses in the quiet-Sun internetwork and network lanes.
\end{abstract}

Key words. Sun: chromosphere - Sun: magnetic fields - stars: chromospheres

\section{Introduction}

Upper chromospheric heating in the Sun has been an important topic for many years (e.g., Schatzman 1949) and it was found that a chromospheric plateau at temperatures of around $6000-7000 \mathrm{~K}$ is needed for explaining the observations of line and continuum emissions by non-active solar surface features (e.g. Athay 1966). These temperatures are higher than those predicted by radiative equilibrium models and require substantial non-radiative energy dissipation. Empirical modeling of the chromospheric plateau structure was carried out using Skylab UV observations (e.g. Vernazza et al. 1981, hereafter VAL; and later Fontenla et al. 1991, 1993, hereafter FAL). However, the lack of understanding of the chromospheric heating physical mechanism made the task of interpreting the observations extremely difficult as is shown recently by Fontenla et al. (2007a, hereafter $\mathrm{FBH}$ ).

Since it was known that convective motions of the upper photosphere carry enough energy to account for the chromospheric losses, shock waves were first thought to be responsible for heating the quiet-Sun chromospheric regions (e.g. Osterbrock 1961). Very detailed simulations were carried out by Carlsson \& Stein 1992) who argue the solar chromosphere is due to transient shock heating. However, recent papers point out that such shocks are not consistent with the amount and pervasive nature of the heating (e.g. Fossum \& Carlsson 2005, 2006; Judge et al. 2003). Strong shocks, if present, would produce the disappearance of the emissions during the waves expansion phase and this is not observed (Carlsson 2007).

Fontenla et al. (2007b) show for the quiet-Sun a correlation of the UV intensity distribution with magnetic fields that indicates a relationship between chromospheric heating and magnetic fields. This relationship was observed before for somewhat stronger fields in plage and network (e.g. Howard 1959; and Milkey 1970). It has been suggested that impulsive nano-flares related to magnetic reconnection (e.g. Sturrock 1999) may play a role. The observations show numerous fast brightening in the Sun but they are not sufficiently frequent (see Aschwanden et al. 2000) to explain the ubiquitous and persistent UV emission of the solar chromosphere (e.g. Carlsson 2007).

Steady electric currents or continuous dissipation of MHD waves have also been suggested mechanisms for chromospheric heating (e.g. Alfven 1947, Uchida \& Kaburaki 1974; Campos $\&$ Mendes 1995). However, because of the large electrical conductivity of solar plasma strong variations of the magnetic field and extremely localized electric currents are required to produce enough energy dissipation. The paper by Socas-Navarro (2007) shows that even in a sunspot, while resistive current dissipation contributes to heating the sunspot chromosphere, it is not the dominant factor. 
Goodman (1997) and Kazeminezhad \& Goodman (2006) note that, because of electrons high magnetization, the Pedersen conductivity is much lower than parallel conductivity in the partially ionized chromospheric plasma and invoke dissipation of electric currents. However, despite of the lower electron conductivity the protons and heavy ions are unmagnetized and highly conductive resulting in a Joule dissipation rate that remains relatively low for expected electric currents in the quiet-Sun (Fontenla 2005). Also, heating due to Pedersen resistivity is not consistent with the steep onset of chromospheric heating above the low chromospheric layers whose temperatures are near radiative equilibrium.

In this paper we propose a mechanism for chromospheric heating due to the rapid onset of the Farley-Buneman (FB) instability marking the start of the upper chromosphere and strong magnetic heating. The FB instability drives heating events in the E layer of the Earth ionosphere (Farley 1963), an environment that in some respects is similar to the solar chromosphere. Fontenla (2005) showed that the conditions for the instability were met in a quiet-Sun atmosphere model considering very small magnetic fields and p-mode oscillations. Here we show that considering recent measurements of the magnetic field the convective overshoot motions in the lower chromosphere are expected to drive the FB instability.

The observations of Hanle effect (Trujillo Bueno et al. 2004, 2006) and recently of the Zeeman effect from Hinode are consistent with horizontal fields of 30 to $160 \mathrm{G}$ in the quiet-Sun granulation (depending on assumptions about filling factor, see Orozco Suárez et al. 2007), that are stronger than those assumed by Fontenla (2005). Our calculations use the FBH model and show that the low-chromosphere is close to radiative equilibrium. The rapid rise in electron temperature to the upper chromosphere plateau in the model allows this model to simultaneously explain the chromospheric CO lines, UV continuum, and the radio observations. Our calculations show that the radiative losses in the FBH model rapidly increase up to those in the upper chromosphere where large departures from radiative equilibrium exist. In the following we discuss the chromospheric parameters that are critical for triggering of the FB instability and estimate the amount of energy that can be released.

Our analysis shows that convective overshoot motions are expected to trigger the FB instability at the pressures where the FBH semi-empirical model display the steep temperature rise from lower to upper chromospheric temperatures. We also show that the energy available at these pressures can account for the upper chromospheric radiative losses.

\section{Inter-network solar atmospheric model}

We consider here model SRPM 306 of the quiet-Sun low chromosphere which has the same temperature vs. height as model SRPM 305 that was described by FBH. The only difference is that SRPM 306 includes full non-LTE radiative transfer calculations for the neutral and singly ionized stages of $\mathrm{C}, \mathrm{Na}, \mathrm{Mg}, \mathrm{Al}$, $\mathrm{Si}, \mathrm{S}, \mathrm{Ca}$ and neutral $\mathrm{He}$, in addition to the previously considered. (Calculations for neutral stages of $\mathrm{H}, \mathrm{C}, \mathrm{N}$, and $\mathrm{O}$ were carried for SRPM 305.) Figure 1 shows the temperature, height, and pressure profiles from SRPM 306. This one-dimensional model represents a weighted average over sub-resolution horizontally fluctuating properties in inter-network regions. The weights of the components correspond to their effects on the emitted radiation at the spectral bands considered in FBH.

Because of the temperature and density values, in the lower- and upper-chromospheric layers the elements with low

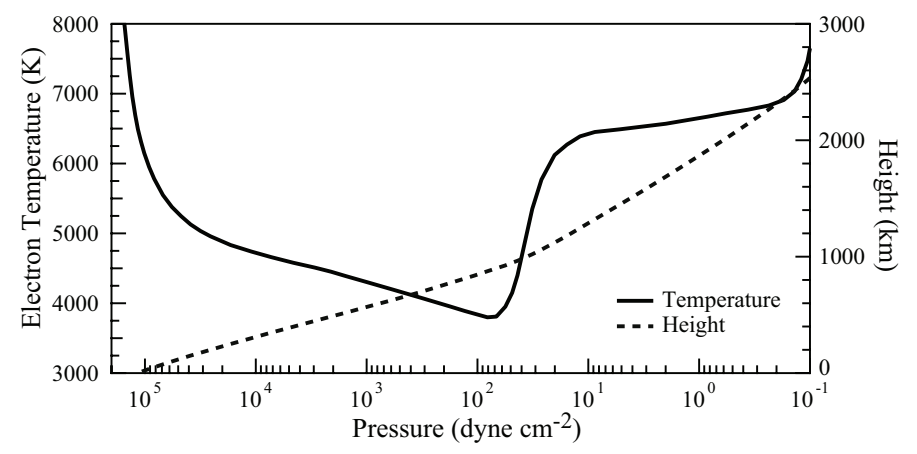

Fig. 1. Temperature and height structure of the chromospheric model SRPM 306.

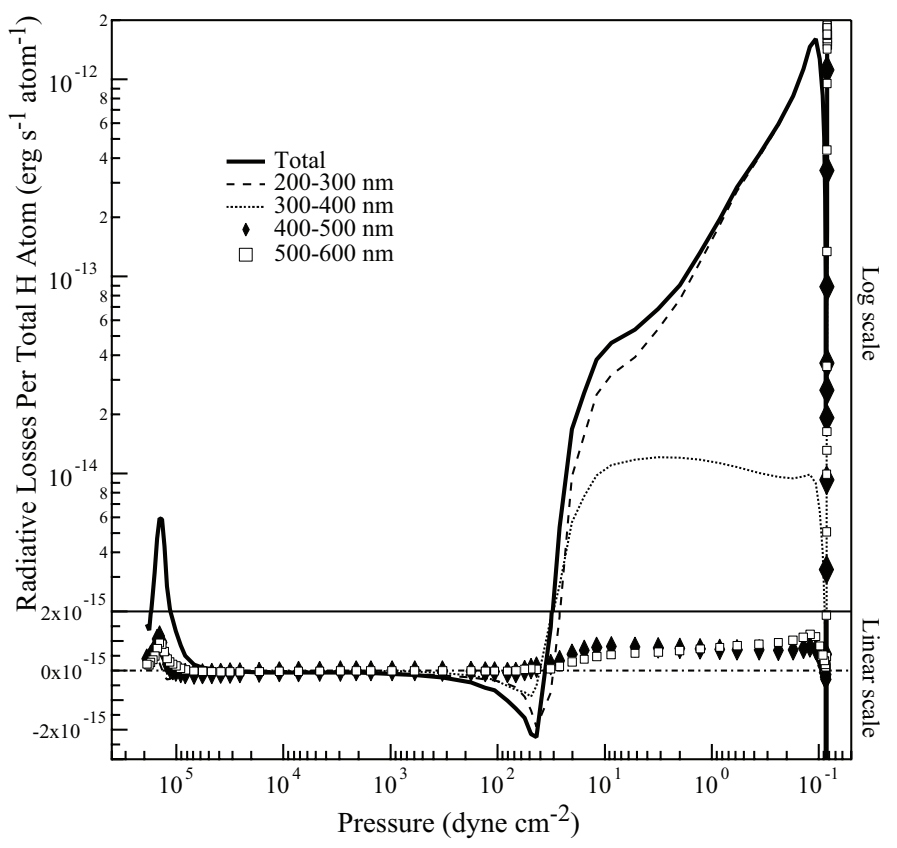

Fig. 2. Ratio of radiative losses to hydrogen density computed for model SRPM 306.

first-ionization-potential (e.g., $\mathrm{Si}, \mathrm{Mg}, \mathrm{Fe}, \mathrm{Ca}$, etc.) are predominately singly ionized. This characteristic of the solar chromosphere is independent of the details of the model. Our calculations of the net radiative losses indicate that, as suggested by Rutten (2007), the atmosphere remains very close to radiative equilibrium up to the pressure at which the temperature rises steeply to the chromospheric plateau. While there is no substantial energy dissipation in the low chromosphere, very important non-radiative energy dissipation is needed immediately above the temperature minimum to account for the observations. This implies a sudden turn-on of the chromospheric heating that is expected to depend on the gas pressure and magnetic field. Figure 2 shows the ratio of radiative losses to total hydrogen density for several spectral bands and the total for wavelengths between $300 \mathrm{~nm}$ and 3 micron. However, the model SRPM 306 assumes LTE for important species such as Fe I and II and Ni I and II which overestimates the upper chromospheric radiative losses. Further refinements will yield more reliable details on the upper chromospheric losses but will not change the conclusions in this paper.

Figure 2 shows: 1) the near radiative equilibrium character of the low chromosphere; and 2) that radiative losses are important only in the photosphere (at pressures larger than 


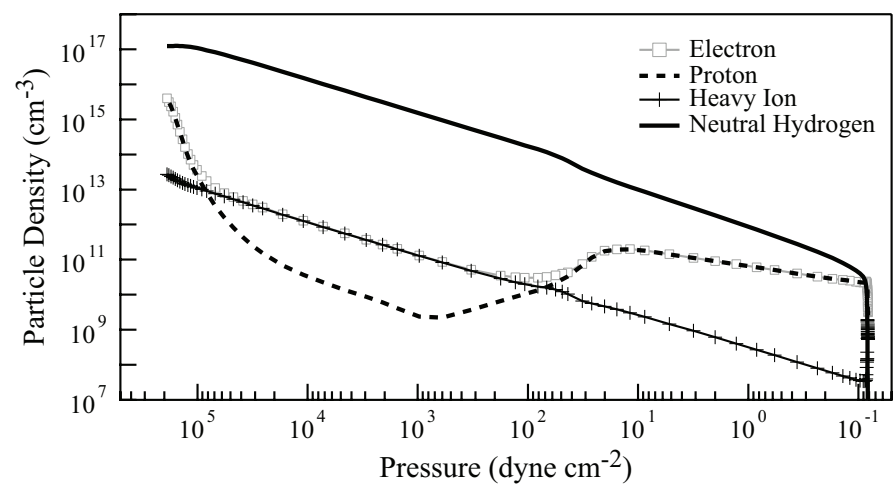

Fig. 3. Particle densities in model SRPM 306. Over the low chromosphere heavy ions dominate the positive charge.

$\sim 5 \times 10^{4} \mathrm{dyne}^{-2}$ ) and in the upper chromosphere. The photospheric radiative losses per $\mathrm{H}$ atom are not as large as those in the chromosphere, but the losses per unit volume are much larger in the deep photosphere. For wavelengths shorter than $400 \mathrm{~nm}$ the losses per $\mathrm{H}$ atom are large in the upper chromosphere.

\section{The plasma ionization}

The chromospheric gas is essentially composed by neutral $\mathrm{H}$ and $\mathrm{He}$, and charged particles are not very abundant (e.g. Fontenla 2005). Another important characteristic of most models, including SRPM 306, is that in the lower chromosphere and near the temperature minimum the proton contribution to the charge balance is small and the positive charge is supplied by the singly ionized heavy metals. Because of the temperature rise, in the upper chromospheric plateau protons are the main positive charge but their density is still much smaller than the neutral $\mathrm{H}$ particle density. Figure 3 shows the electron, proton, and ion densities in model SRPM 306 and illustrates this point.

This property of the positive charge contributions turns out to be very important because in the low chromosphere the dominance of heavy ions on the charge balance produces a very low value of the average ion acoustic velocity. The isothermal ion acoustic velocities (e.g. Farley 1963 ) for protons, $C_{\mathrm{sp}}$, ions, $C_{\mathrm{si}}$, and the average, $C_{\mathrm{s}}$, are defined by the following equations

$C_{\mathrm{sp}}=\sqrt{\frac{k T_{\mathrm{e}}}{m_{\mathrm{p}}}}$,

$C_{\mathrm{si}}=\sqrt{\frac{k T_{\mathrm{e}}}{m_{\mathrm{i}}}}$,

$C_{\mathrm{s}}=\sqrt{\frac{n_{\mathrm{e}} k T_{\mathrm{e}}}{n_{\mathrm{p}} m_{\mathrm{p}}+\sum n_{\mathrm{i}} m_{\mathrm{i}}}}$.

Figure 4 shows the proton and ion acoustic velocities for a typical positive ion ( $\mathrm{Si}$ II) and the average ion acoustic velocity as function of pressure in model SRPM 306. Note that the average ion acoustic velocity is much smaller than the gas sound speed, which is well represented by the proton ion acoustic velocity, in the entire low chromosphere as a consequence of the dominant contribution of heavy ions to the positive charge. The heavy ion isothermal sound speed at the temperature minimum layer ranges from $\sim 1.0$ to $\sim 1.5 \mathrm{~km} \mathrm{~s}^{-1}$ (for Fe II and Si II respectively); and at photospheric layers ranges from $\sim 1.5$ to $\sim 2.0 \mathrm{~km} \mathrm{~s}^{-1}$.

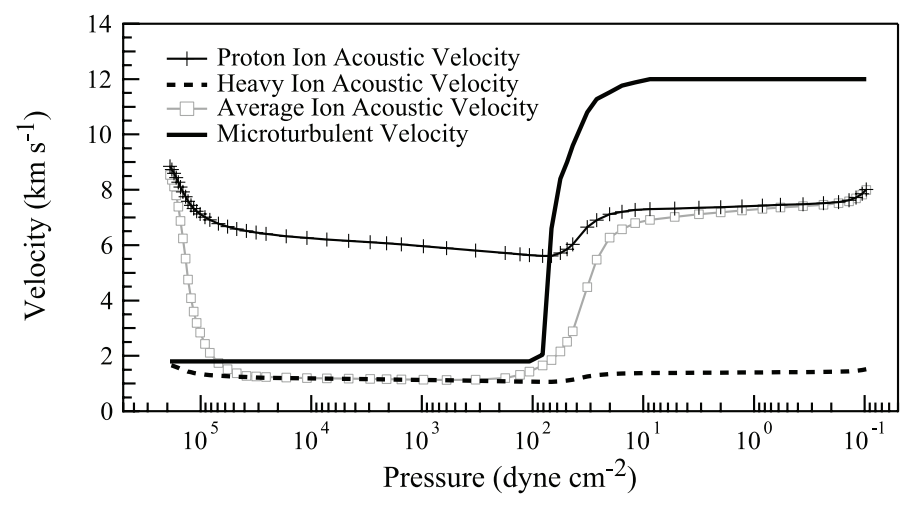

Fig. 4. Ion acoustic and microturbulent and velocities in model SRPM 306. Microturbulent velocities are discussed in Sect. 3.

We show later that the low ion-acoustic velocity reduces the minimum motion velocities needed to trigger the FB instability in the low chromosphere to the point where convective overshoot motions are able to drive the plasma to a FB unstable regime. Figure 4 also shows the microturbulence velocity that will be will be discussed in the next section.

\section{Convective velocities and microturbulent line broadening}

The FB instability is driven by the component of the neutral particles motion in the direction perpendicular to the magnetic field. For the instability to occur cross-field motions faster than the ion acoustic velocity are needed. This condition is met by the turbulent convective velocities in the photospheric granulation (in excess of $2 \mathrm{~km} \mathrm{~s}^{-1}$, e.g. de Jager \& Neven 1968; and Stein $\&$ Nordlund 2000) because of the low value of the ion acoustic velocity for the heavy species that carry the positive charge above the top of the photosphere (pressure $\lesssim 5 \times 10^{4} \mathrm{dyne}^{-2}$ ). Stein \& Nordlund (2000) have performed time-dependent threedimensional simulations of the solar convection. In their simulations the RMS vertical velocities decrease above the photosphere but the RMS horizontal velocities remain above $2.5 \mathrm{~km} \mathrm{~s}^{-1}$ at the top of the domain. At the photosphere both vertical and horizontal RMS velocities are around $2.8 \mathrm{~km} \mathrm{~s}^{-1}$. They obtained an RMS velocity value of about $3 \mathrm{~km} \mathrm{~s}^{-1}$ at the top of their simulation domain (mean pressure $\sim 5 \times 10^{3}$ dyne $\mathrm{cm}^{-2}$ ). The vertical velocities at the top of the simulation domain have a maximum magnitude of about $1 \mathrm{~km} \mathrm{~s}^{-1}$ and the horizontal 6 or $7 \mathrm{~km} \mathrm{~s}^{-1}$. However, Stein \& Nordlund (2000) and similar 3-dimensional simulations of convection dynamics do not reach the layers at which the temperature minimum occurs. In general, numerical simulations of the low chromosphere are not yet physically consistent with the complexities in:

- solving 3-dimensional radiative transfer accurately for the very complicated spectrum from the lower chromosphere with a multitude of lines of atomic and molecular species;

- departures from LTE in many species and irradiation from the photosphere and corona;

- the upper boundary conditions are affected by the not well known upper layers magnetic fields;

- Unknown effects and heating related to magnetic fields (e.g. the FB instability).

We do not yet know from the theory of convection the velocities and spatial characteristics at the top of the lower chromosphere (pressure $\sim 40$ to 100 dyne $\mathrm{cm}^{-2}$ ) where the strong 
chromospheric heating is triggered. It is clear from the current theory, however, that RMS velocities of at least between 2 and $3 \mathrm{~km} \mathrm{~s}^{-1}$ are expected and that larger values occur at certain locations and times (e.g. strong downflows in dark intergranular lanes).

Observational constraints on convective and turbulent motions are provided by the spectral line shift and broadening. Near solar disk center, the absorption lines in the photosphere and low chromosphere display unresolved line shifts and a microturbulent non-thermal broadening that slightly decreases from $\sim 2.4$ in the photosphere to $\sim 1.8 \mathrm{~km} \mathrm{~s}^{-1}$ in the low chromosphere. We base these estimates of turbulent vertical velocity on many photospheric and lower chromospheric lines shown in $\mathrm{FBH}$, e.g. the $\mathrm{CN}$ band around $388.4 \mathrm{~nm}$ and the $\mathrm{CO}$ lines at 4.4554 micron. The microturbulent velocity found at the photosphere is consistent with the granular convective velocities derived from the simulations mentioned above. At $\sim 2$ " resolution, the observed non-thermal photospheric line broadening is explained by the motions in unresolved granular structures. However, in the lower chromosphere the observations indicate larger vertical velocities than those at the top of the Stein \& Nordlund simulation domain. This may be due to inaccuracies of the simulation near the upper boundary or to physical processes not yet accounted for. Horizontal velocities are much harder to estimate from observed line widths. Only center-to-limb behavior can shed some light on them but detailed observations relevant to this issue are not published. In any case such observations would be very difficult to interpret because of the effects of uncertainties in the models, the temporal and spatial fluctuations, and the extent of the line formation region.

The detailed geometry of the magnetic fields and velocities in the low chromosphere is not well known. It is not yet clear what the cross-field component of the velocity is despite recent work on magneto-convection simulations because these have the same limitations mentioned above. Thus, in this paper we conservatively assume for the low chromosphere a velocity value of $1.8 \mathrm{~km} \mathrm{~s}^{-1}$ in the cross-field direction.

Turbulent motions in the upper chromosphere are much less understood. There are abundant observations of strong lines (e.g. from $\mathrm{H}$ Balmer lines and from $\mathrm{Ca}$ II H and $\mathrm{K}$ lines) that display motions at velocities of $\sim 10 \mathrm{~km} \mathrm{~s}^{-1}$ and larger above the limb, for instance in spicules. However, it is not clear how these develop and whether lower altitude similar features are present in the inter-network jungle of unresolved fine structures. Even at the highest resolution available the spectral lines display large non-thermal broadening that corresponds to near sonic or slightly supersonic velocity. The complicated line formation and the unresolved small spatial structure, shown by rare high quality images, make it impossible to provide model-independent diagnostic in the upper chromosphere. Whether the microturbulent non-thermal line broadening corresponds to motions within this fine structure of hundreds of km scale or at smaller scales (even at mean free path or ion gyroradius scale) is still unsettled.

Upper chromospheric 3-dimensional simulations that consider propagation of MHD waves have been performed (e.g. Bogdan et al. 2003), but these do not consider the multicomponent plasma process we consider here and assume a simplified energy equation. One-dimensional numerical simulations of shocks consider NLTE radiative transfer and radiative losses but ignore magnetic effects and lead to expectation of very supersonic velocities (e.g. Carlsson \& Stein 1992). However, as noted above the observations of UV line emissions do not agree with these simulation predicted behavior (Carlsson 2007). Theoretical adiabatic arguments indicate increasing values of

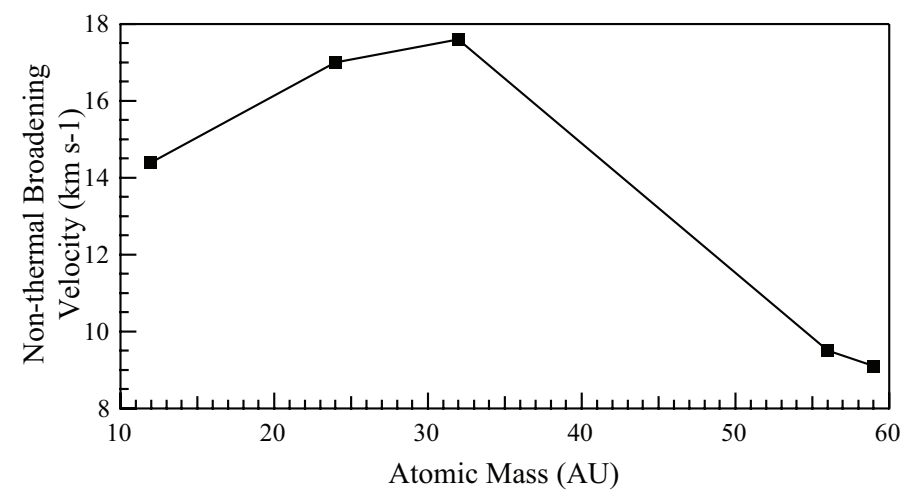

Fig. 5. Fit to observed chromospheric line broadening velocities (see text).

velocity as the inverse of the square root of the average density changes with height. However, in a non-adiabatic case, radiative losses tend to reduce any increases of the motions velocity. It is very difficult to evaluate theoretically the net result on the turbulent velocity of these competing effects because of the issues we discussed above in reference to the convection simulations.

Previous one-dimensional steady-state chromospheric models (e.g. VAL, FAL) used a simplistic scheme for assigning a non-thermal broadening velocity in the upper chromosphere in order to compute line profiles with non-LTE radiative transfer methods. This procedure gradually increases microturbulent velocity throughout the upper chromosphere and reaches near $12 \mathrm{~km} \mathrm{~s}^{-1}$ at the base of the chromosphere-corona transition region. Observational constraints are available at the transition region layers but such constraints have not been extensively explored at the base of the chromospheric plateau. Line broadening observational studies for these layers would likely be inconclusive because of the model dependence of the computed profiles, the one-dimensional steady-state nature of the available chromospheric models, and the complexity of the emitted line radiation formation. However, as we show next the observations provide clear indications that the broadening velocities previously used in models are too low for explaining UV line emission profiles.

We use SUMER observations (Curdt et al. 2001) in order to estimate microturbulent velocities from a number of very weak spectral lines which form at the base of the upper chromosphere. Figure 5 shows the values of non-thermal velocities found by fitting simple Gaussians to several lines and taking into account the SUMER instrument resolution and thermal broadening. It is difficult to ascertain whether a steep variation of the microturbulence over the region of formation of these lines or over the spatial and temporal resolution of the data may be responsible for the variations observed. We therefore conclude from these data that non-thermal broadening velocities of emission lines formed near the base of the upper chromosphere range from $\sim 9$ to $\sim 18 \mathrm{~km} \mathrm{~s}^{-1}$. This shows that the microturbulent velocity at the base of the chromospheric plateau was underestimated in previous semi-empirical models that used the VAL/FAL scheme and may depend on the species considered.

To verify this finding Fig. 6 shows a portion of the SUMER spectrum representative of the formed at the base of the chromospheric plateau and demonstrates the point that the scheme used in VAL and FAL to assign line broadening microturbulent velocities produces profiles that are too narrow when compared with the observed spectrum. However, agreement is attained when additional convolution with a Gaussian profile is applied that accounts for the instrument resolution and with an additional 
non-thermal Doppler broadening velocity of $\sim 10 \mathrm{~km} \mathrm{~s}^{-1}$ ). This result is consistent with the results shown in Fig. 5 and our microturbulent velocity in Fig. 4.

In summary, the data and analysis here is consistent with a sudden rise of the broadening velocity, similar to that of the temperature, that reaches a plateau in the upper chromosphere that is analogous to that in the temperature profile. We consider the microturbulence velocity, $V_{\mathrm{t}}$, discussed above to be the responsible for the triggering of the FB instability. In the low chromosphere and photosphere this velocity is consistent with the inferred from convection simulations and in the upper chromosphere it is consistent with observed fine scale motions and non-thermal broadening.

\section{Collision rates and plasma frequency}

The FB instability occurs in the solar chromosphere because electrons become strongly magnetized just above the photosphere while heavy ions and protons remain unmagnetized for the whole chromosphere and only at its very top they become magnetized. Particle magnetization can be described as the ability of charged particles to perform Larmour gyrations as they are subject to collisions that interrupt their motion. Of course magnetization is also important for determining the Pedersen and Hall conductivities in a partially ionized plasma. However, the Pedersen conductivity is affected by the magnetization in a continuous way. As discussed by Fontenla (2005) Pedersen resistivity produces low-power energy dissipation over all chromospheric layers, whereas the FB instability has a minimum threshold and cannot occur below the critical value. Once above it the FB instability rapidly grows becoming non-linear, and substantially dissipates energy to produce the observed steep electron temperature rise characteristic of the upper chromosphere. Figure 7 shows the total collision rates for electrons, protons, and a typical heavy ion (the figure shows Si II because it is one of the most abundant) along with the plasma frequencies as a function of pressure in the model SRPM 306. Note that the collisions include those between charged particles and neutral $\mathrm{H}$ and include elastic and inelastic rates. The collision rates with neutral $\mathrm{H}$ are based on approximate cross-sections.

It has been proven difficult to determine the lower chromospheric structure of the quiet-Sun network features. Observations show that these features correspond to stronger magnetic fields but comparison of the their thermal stratification with that of the model SRPM 306 are difficult because of the lack of published spectral observations discriminating between these features. FBH reports that there are no significant differences between inter-network and network observations of line profiles for spectral lines formed at pressures larger than $\sim 10^{3}$ dyne $\mathrm{cm}^{-2}$. The observations of absorption lines whose line centers form at lower pressures display spatial variations which indicate that the temperature rise to the upper chromospheric plateau starts at a larger pressure with increasing magnetic field. As consequence of the change in the location where the temperature starts increasing, the minimum value of the temperature also increases as the magnetic field increases.

The present paper explores these effects by assuming the same low chromospheric structure for the internetwork and network and examines how an increasing magnetic field leads to the triggering of the FB instability at higher pressures. The FB instability threshold analysis in the next section permits us to quantify this effect and produces values of the pressure at the base of the upper chromosphere that can be used to construct better models for the network.
Our present broad brush approach is consistent with the hypothesis that departures from radiative equilibrium are small in the low chromosphere up to the point where the strong chromospheric heating suddenly starts. This approach neglects the effects of the lower chromosphere being irradiated by the upper chromosphere and dissipation of magnetic energy in the lower chromosphere. We assume here that these effects are small and that SRPM 306 is still a good description for the layers below the temperature minimum for any magnetic fields below $\sim 1000 \mathrm{G}$. It is further assumed that convective velocities and the average structure of the lower chromosphere are not significantly modified by such weak magnetic fields. Specifically, in our analysis it is only the intensity of the magnetic field that determines the pressure threshold of the FB instability through the change in particle magnetization.

\section{Magnetic fields and FB instability onset}

Evidence of the existence of magnetic fields in the range of 30-160 G in the quiet-Sun internetwork comes from observations of the Hanle effect (Trujillo Bueno et al. 2004, 2006). Hinode measurements by Orozco Suárez et al. (2007) recently confirmed this using the Zeeman effect. The observations show the prevalence of a distribution of horizontal magnetic fields over the quiet-Sun inter-network, as well as the previously known stronger and more organized fields in the network and active regions.

Figure 8 shows the magnetization, $M=f_{\mathrm{L}} / v$ (i.e. the Larmour frequency divided by collision rate) as a function of pressure in the model for three charged particles. This figure shows that electrons become strongly magnetized just above the photosphere. Also, the figure shows that protons and ions remain unmagnetized (magnetization less than or about unity) for the whole chromosphere and only become magnetized at the chromosphere-corona transition region.

The velocity of the mostly neutral gas colliding with plasma provides an electric field in the center-of-mass frame, $E=$ $(U \times B)$, that drives the FB instability. Vertical motions in horizontal field regions (e.g. the "neutral lines" of longitudinal field magnetograms) and horizontal motions in vertical field regions (e.g. buffeting of pores by the external medium) are readily observed. Other cases involve horizontal motions at large angle within horizontal magnetic field regions. We do not study any particular case and assume that the cross field velocity given in Fig. 4 as the driving cross field velocity, $U=V_{\mathrm{t}}$.

Figure 9 shows the FB instability threshold, $R$, for both protons and the selected heavy ion. Values of $R$ larger than one trigger the instability. $R$ is given by the formula (Farley 1963)

$R=\frac{U}{(1+\psi) C_{\mathrm{s}}}$

where $U$ is the convective or turbulent velocity, $\psi$ is a parameter related to the particle magnetization and $C_{\mathrm{s}}$ is the ion acoustic speed derived from the electron temperature and the average ion mass (see Fontenla 2005, and references therein for details).

Figure 9 shows curves for the parameter $R$ for protons and heavy ions ( $\mathrm{Si}$ II is shown) in the model SRPM 306 as a function of magnetic field strength. Because $U>C_{\text {si }}$ over the whole chromosphere the location of the FB threshold $(R=1)$ for heavy ions is determined by $\psi$ and becomes reached at different gas pressures depending on the magnetic field strength. Protons do not reach the FB threshold in the lower chromosphere because of the small value of $U$ in these layers. However, protons reach 


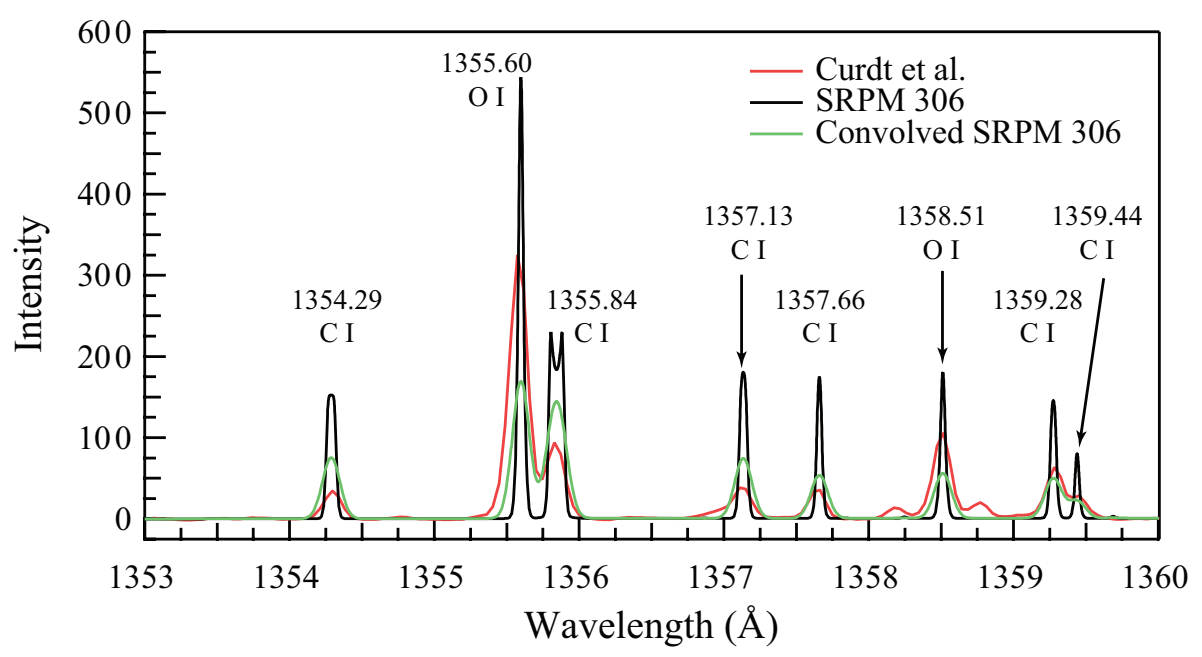

Fig. 6. Observed, computed using VAL/FAL scheme, and convolved profiles of emission lines that form in the chromospheric plateau.

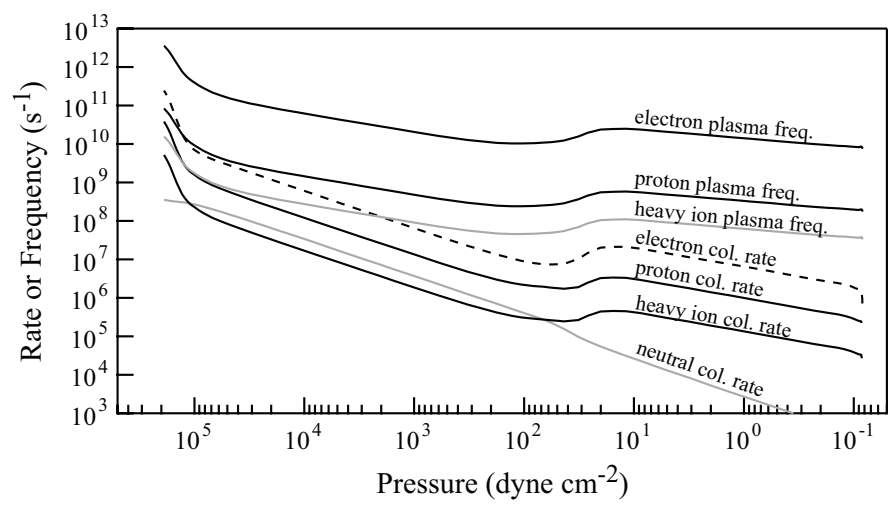

Fig. 7. Collision rates and plasma frequencies in the model.

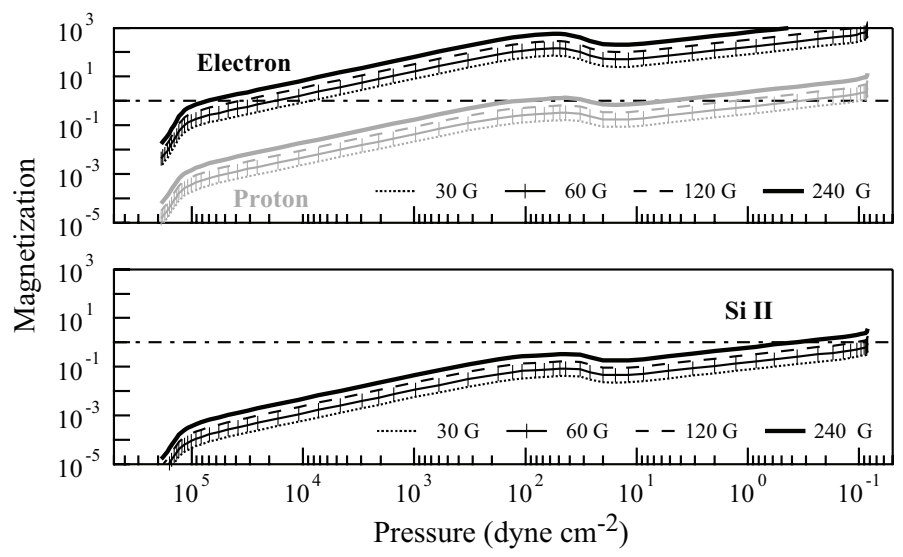

Fig. 8. Particle magnetization in the model.

the FB instability threshold in the upper chromosphere where $U>C_{\text {sp. }}$.

Because in lower chromospheric layers the positive charge is largely dominated by the heavy ions the small value of $U$ in the low chromosphere is larger than the average ion sound speed (see Fig. 4) and the heavy ions curve correctly describes the overall FB instability threshold. In the upper chromosphere protons establish the overall FB threshold because the proton density is larger than the heavy ion density and the average ion sound speed becomes equal to the proton ion sound speed (see Fig. 4).

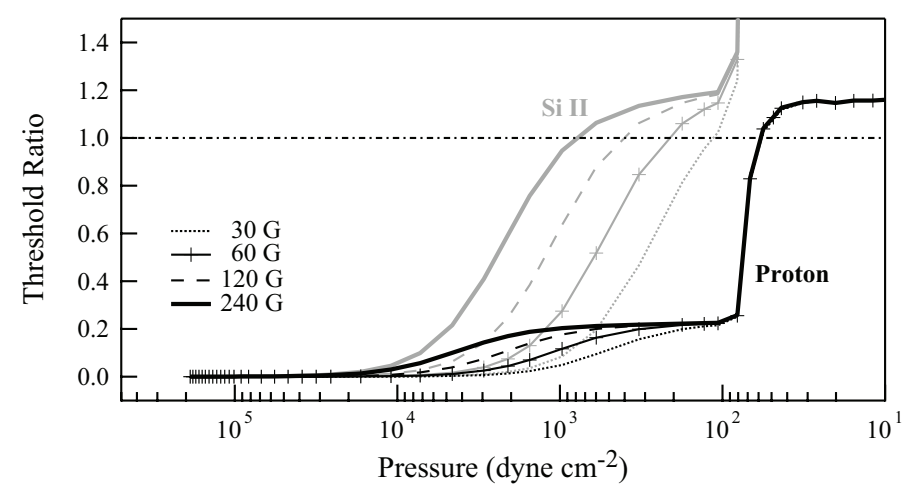

Fig. 9. Ratio corresponding to the FB instability criteria in the model.

The fast turbulent velocities in the upper chromosphere are well established from observations but it remains uncertain how they are generated. It is possible that they could be produced by MHD wave propagation in the form of fast, Alfven, or slowmode waves. However, because the transition between small and large non-thermal broadening velocities corresponds to the rapid temperature increase, it is probable that the onset of these large velocities relates to the primary chromospheric heating mechanism that not only heats electrons but also accelerates and transports upwards heavy particles.

\section{Energy flux at the instability onset}

The charged particle motions in the chromospheric layers are affected by the balance between magnetic forces and collisional forces with the neutral gas. The plasma beta provides a measure of the relative importance of gas pressure (collisions) and Lorentz (magnetic) forces in a plasma. In the partially ionized lower chromosphere we distinguish between the total gas beta and the ionized particles beta because the charged particles motion is directly affected by magnetic fields but not the bulk of the gas since neutrals dominate and are unaffected by the field. The plasma beta curves in Fig. 10 show that in the upper chromosphere the total gas plasma beta is small. As a result one expects that the magnetic forces indirectly dominate the neutral gas motions due to its collisions with the charged particles. Instead, in the lower chromosphere magnetic forces are small compared to pressure forces so that the neutral gas pressure and dynamics are the dominant processes and drive the charged particles dynamics 


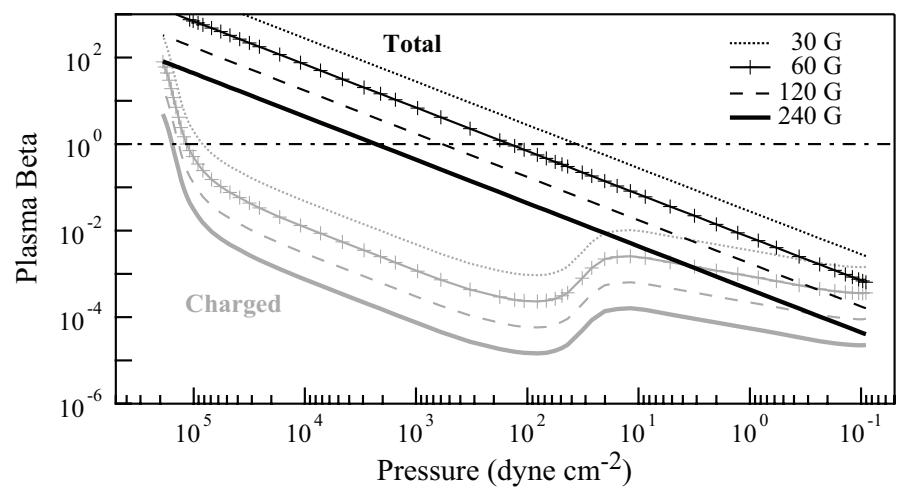

Fig. 10. Plasma beta in the model.

as well. Comparing Figs. 9 and 10 we conclude that, for weak magnetic fields in the lower chromosphere the FB instability criteria is met while the plasma beta is larger than one. At a field of $120 \mathrm{G}$ the FB instability would trigger at the location where plasma beta is one, and for stronger fields the onset of the FB instability occurs at plasma beta less than one.

The energy flux carried by the gas at the instability onset can be estimated from the enthalpy and kinetic energy fluxes as follows

$F_{\text {hydro }}=F_{\text {enth }}+F_{\text {kin }}=U\left(\frac{5}{2} p+\frac{\rho}{2} U^{2}\right)$.

In addition, the magnetic component of the energy flux can be substantial depending on the magnetic field. We consider here fast-mode waves driven by vertical convective motions and the magnetic energy flux can be estimated by the Pointing vector as follows

$F_{\mathrm{mag}}=\frac{E \times B}{8 \pi} \simeq \frac{(U \times B) \times B}{8 \pi} \simeq U p_{\mathrm{mag}}$

where $p_{\text {mag }}$ is the magnetic pressure.

In Table 1 we show the values of the gas parameters at the FB instability onset for the various magnetic field strengths. We note that the available total energy flux for each magnetic field strength is consistent with the estimated radiative losses from the corresponding solar surface feature; in all cases the energy fluxes are much larger than those needed to heat the corona and transition region, and to power the solar wind. The energy flux estimates in Table 1 are only upper limits because part of the available upward energy flux may be reflected by the upper layers. The simple arguments in this paper do not permit us to draw definite conclusions on the reflection and net energy flux but we consider it likely that most of the thermal energy would be actually dissipated in the chromosphere and directly feed the FB waves and radiative losses, but with part of the magnetic energy flux reflected back. It is likely that most of the upward kinetic energy flux will also return but it would be dissipated in the chromosphere as the material falls back.

For stronger magnetic fields than those considered here it is unlikely that the model SRPM 306 would apply. Important changes would occur in the convective motions and the energy balance of the low chromosphere and thus we do not include strong magnetic fields in the present study.

\section{Possible observational diagnostic}

Global scale neutral winds in the Earth's ionosphere transfer momentum to ions in collision dominated regions. The ions driven across magnetic field lines by the neutral winds produce electric fields of few to several $\mathrm{mV} \mathrm{m}^{-1}$ (Peymirat et al. 2002) that drive the FB instability. These electric fields can be measured in situ (e.g., Coley et al. 1994).

In the Sun, the granular motions responsible for the neutral "winds" that we believe drive the FB instability occur mainly at granulation scale which is difficult to observe from the ground. Until recently only numerical simulations of convection were able to unequivocally show that motions of velocities such as we consider are present within granules. Nordlund et al. (1997) shows that "already at modest numerical resolution the simulations account for almost all velocities contributing to the line shape". Magneto-convection simulations (Vögler et al. 2005) display motions across the magnetic field that correspond to those we discuss. However, these simulations use initial and boundary conditions that may not yet be realistic because they assume purely vertical magnetic field at the top and bottom boundaries and they do not yet include realistic tensor electric conductivity with consideration of parallel, Pedersen, and Hall components.

The recent observations by Hinode are able to not only resolve the granulation but also establish the geometry of the relatively strong horizontal magnetic fields that occur near the edges of the granules. According to these observations flows at steep angles with the magnetic field are likely in the photosphere. Cross-field flows with velocities of $1.8 \mathrm{~km} \mathrm{~s}^{-1}$ and a magnetic field strength of $30 \mathrm{G}$ would yield an electric field of $5 \mathrm{~V} \mathrm{~m}^{-1}$ in the frame of the neutral gas. Such fields are expected to have spatial-scales smaller than granular size and would be smeared by the oscillating fields in the FB instability in addition to the usual Stark and Van der Waals broadening fields. Consequently, the electric field produced by the cross-field neutral flow is too small for direct observation using Stark effect (see Casini \& Foukal 1996) but fully adequate to trigger the FB instability.

Since it does not seem feasible to directly observe the electric fields that drive the FB instability at the top of the low chromosphere a better diagnostic would be the simultaneous observation of velocity and magnetic field vectors within the granular scale and at the heights where the FB instability is triggered. The $\mathrm{Na}$ I D line centers form close to the temperature minimum and thus they could provide a useful diagnostic but their relatively large collisional broadening may be a problem. The deep CO lines near 4.66 micron, especially towards the limb, may provide good diagnostic if they can be observed (from space) at spatial, spectral, and temporal resolution good enough to resolve the intragranular flows. Simultaneous vector magnetic fields are also needed for providing the complete data needed to compute the values of $E=v \times B$ that would drive the FB instability.

These measurements could be carried by future instrumentation and would unambiguously characterize the relevant quantities. For now, only numerical simulations of magneto-convection with realistic magnetic and velocity fields and considering the full plasma effects can produce more evidence of the FB onset. Moreover, PIC ("particle in cell") numerical simulations such as those by Otani \& Oppenheim (2006) can be used to assess not only the onset but also the further non-linear development of the FB instability in the solar chromosphere. Such simulations can supply predictions about which observations are best to confirm or negate the scenario we propose.

\section{Conclusions}

This paper proposes a novel chromospheric heating mechanism that relies on the turbulent motions of the low chromosphere that 
Table 1. Gas parameters and energy flux at the FB onset.

\begin{tabular}{|c|c|c|c|c|c|c|c|c|}
\hline Code & $\begin{array}{c}\text { Pres. } \\
\text { dyne } \mathrm{cm}^{-2}\end{array}$ & $\begin{array}{c}\text { Height } \\
\text { km }\end{array}$ & $\begin{array}{c}\text { Temp. } \\
\text { K }\end{array}$ & $\begin{array}{l}B \\
\mathrm{G}\end{array}$ & $F_{\text {enth }}$ & $\begin{array}{l}F_{\text {kin }} \\
\mathrm{erg} \mathrm{cm}^{-}\end{array}$ & $\begin{array}{l}F_{\mathrm{mag}} \\
-2 \mathrm{~s}^{-1}\end{array}$ & $\overline{F F_{\text {tot }}}$ \\
\hline $\bar{B}$ & 110 & 840 & 3850 & 30 & $4.8 \mathrm{e} 7$ & $3.5 \mathrm{e} 6$ & $6.4 \mathrm{e} 6$ & $2.9 \mathrm{e} 7$ \\
\hline C & 180 & 770 & 3960 & 60 & $7.9 \mathrm{e} 7$ & $6.7 \mathrm{e} 6$ & $2.6 \mathrm{e} 7$ & $6.4 \mathrm{e} 7$ \\
\hline D & 330 & 700 & 4080 & 120 & $1.5 \mathrm{e} 8$ & $1.5 \mathrm{e} 7$ & $1.0 \mathrm{e} 7$ & $1.8 \mathrm{e} 8$ \\
\hline $\mathrm{E}$ & 600 & 625 & 4200 & 240 & $2.7 \mathrm{e} 8$ & $3.6 \mathrm{e} 7$ & $4.1 \mathrm{e} 8$ & $5.6 \mathrm{e} 8$ \\
\hline $\mathrm{F}$ & 1540 & 520 & 4390 & 480 & $6.9 \mathrm{e} 8$ & $1.1 \mathrm{e} 8$ & $1.6 \mathrm{e} 9$ & $2.0 \mathrm{e} 9$ \\
\hline
\end{tabular}

trigger the FB plasma instability. This instability provides the energy dissipation that feeds the upper chromospheric radiative losses. The most important new insight is that the FB plasma instability is triggered at the top of the lower chromosphere and provides the means to dissipate the motions energy into plasma waves and electron heating. The dissipation mechanism we propose is new in the context of solar physics and is analogous to a phenomenon that has been observed and analyzed in the context of the Earth's ionosphere. However, the mechanism proposed here is not new in regard to the source of the energy, convective motions of the photosphere, which is known to be sufficient to power the chromosphere and upper layers (e.g. Osterbrock 1961).

The mechanism we propose does not require sudden temporal changes of gas pressure and can be considered an almost continuous heating mechanism. The development of the FB instability produces sudden changes and small scale structures in the gas. Fluctuations are also expected to occur in the process as velocities and magnetic fields change but one remarkable property is that the FB instability is triggered regardless of compression or expansion of the gas. Energy from $p$-mode oscillations could also supply energy that is dissipated through the FB instability and may provide a secondary source of energy. The role of the inter-network magnetic field is only to provide a mechanism by which the energy in convective motions is converted into plasma waves and thermal motions of the gas. The FB instability does not require magnetic free-energy to operate.

The consideration of the FB instability onset as a trigger for the dissipation of energy from convective overshoot motions gives a strong constraint on the pressure at which the electron temperature rapidly rises from the atmosphere temperature minimum up to the values found in the chromospheric plateau region. These considerations give a correspondence between the gas pressure, velocity, and magnetic field strength at the FB instability onset from which we derive the amount of energy available for dissipation. This analysis will lead to a set of revised models of the chromosphere very different from the previous (e.g. VAL, FAL) and better constrained by basic physical processes.

Numerical simulations of the FB instability under solar chromospheric conditions are needed to asses the details of this complex process. Although this process has been studied in the Earth's ionosphere E-region its non-linear behavior is still under study (e.g. St.-Maurice 1987, Dimant \& Sudan 1995; Oppenheim et al. 1995, and Otani \& Oppenheim 2006). In particular it is very important to study how the FB waves partition energy between neutrals motions and the heating and acceleration of electrons and ionized species in the non-linear regime.

The temperature and elemental ionization conditions that determine the triggering of the FB instability are similar in most cool stars of solar type. Also the convective motions and overshoot are present in them and thus the FB instability may be responsible for chromospheric heating in many stars. In the quietSun, chaotic magnetic fields appear to be produced by a surface dynamo whose origin is expected to relate to the convective motions. Stronger, larger scale and more organized magnetic fields in the Sun correspond to the solar cycle and are believed to be produced by a global dynamo process. Although in other convective stars the local dynamo is probably similar to that in the Sun, the global dynamo depends on stellar properties of rotation, differential rotation, and meridional flows that may vary widely since they are related to the detailed history of each star.

Acknowledgements. This work was supported by NASA contracts NAS5-97045 and NNX07AO75G at the University of Colorado.

\section{References}

Alfven, H. 1947, MNRAS, 107, 211

Aschwanden, M. J., Tarbell, T. D., Nightingale, R. W., Schrijver, C. J., \& Title, A. 2000, ApJ, 535, 1047

Athay, R. G. 1966, ApJ, 146, 223

Bogdan, T. J., Carlsson, M., Hansteen, V., et al. 2003, ApJ, 599, 626

Campos, L. M., \& Mendes, P. M. V. M. 1995, MNRAS, 276, 1041

Carlsson, M. 2007, in The Physics of Chromospheric Plasmas, ed. P. Heinzel, I.

Dorotovič, \& R. J. Rutten, ASP Conf. Ser., 368, 49

Carlsson, M., \& Stein, R. F. 1992, ApJ, 397, L59

Casini, R., \& Foukal, P. 1996, Sol. Phys., 163, 65

Coley, W. R., Heelis, R. A., \& Spencer, N. W. 1994, JGR, 99, 341

Curdt, W., Brekke, P., Feldman, U., et al. 2001, A\&A, 375, 591

de Jager, C., \& Neven, L. 1968, Sol. Phys., 4, 379

Dimant, Y. S., \& Sudan, R. N. 1995, JGR, 100, 14,605

Farley, D. T. Jr. 1963, JGR, 68, 6083

Fontenla, J. M. 2005, A\&A, 442, 1099

Fontenla, J. M., Avrett, E. H., \& Loeser, R. 1991, ApJ, 377, 712

Fontenla, J. M., Avrett, E. H., \& Loeser, R. 1993, ApJ, 406, 319 (FAL)

Fontenla, J. M., Balasubramaniam, K. S., \& Harder, J. 2007a, ApJ, 667, 1243 $(\mathrm{FBH})$

Fontenla, J., Curdt, W., Avrett, E. H., \& Harder, J. 2007b, A\&A, 468, 695

Fossum, A., \& Carlson, M. 2005, Nature, 435, 919

Fossum, A., \& Carlson, M. 2006, ApJ, 646, 579

Goodman, M. L. 1997, A\&A, 324, 311

Howard, R. 1959, ApJ, 130, 193

Judge, P. G., Carlsson, M., \& Stein, R. F. 2003, ApJ, 597, 1158

Kazeminezhad, F., \& Goodman, M. L. 2006, ApJS, 166, 613

Milkey, R. W. 1970, Sol. Phys., 14, 62

Nordlund, Å., Spruit, H.C., Ludwig, H.-G., \& Trampedach, R. 1997, A\&A, 328, 229

Oppenheim, M., Otani, N., \& Ronchi, C. 1995, GRL, 22, 353

Orozco Suárez, D., Bellot Rubio, L.R., del Toro Iniesta, J.C., et al. A 2007, ApJ, 670, L61

Osterbrock, D. E. 1961, ApJ, 134, 347

Otani, N. F., \& Oppenheim, M. 2006, JGR, 111, A03302

Peymirat, C., Richmond, A. D., \& Roble, R. G. 2002, JGR, 107, 1006

Rutten, R. J. 2007, in The Physics of Chromospheric Plasmas, ed. P. Heinzel, I.

Dorotovič, \& R. J. Rutten, ASP Conf. Ser., 368, 27

Schatzman, E. 1949, AnAp, 12, 203

Socas-Navarro, H. 2007, ApJ, 633, L57

St.-Maurice, J.-P. 1987, JGR, 92, 4533

Stein, R. F., \& Nordlund, A. 2000, Sol. Phys., 192, 91

Sturrock, P. A. 1999, 421, 451

Trujillo Bueno, J., Shchukina, M., \& Asensio Ramos, A. 2004, Nature, 430, 326 Trujillo Bueno, J., Asensio Ramos, A., \& Shchukina, M. 2006, ASP Conf. Ser., 358, 269

Uchida, Y., \& Kaburaki, O. 1974, Sol. Phys., 35, 451

Vernazza, J. E., Avrett, E. H., \& Loeser, R. 1981, ApJS, 45, 635 (VAL)

Vögler, A., Shelyag, S., Schüssler, et al. 2005, A\&A, 429, 335 